\title{
Estado Nutricional de Escolares do Semi-Árido do Nordeste Brasileiro
}

\section{The nutritional status of schoolchildren living in the semi-arid area of northern Brazil}

João B. da Silva ${ }^{1}$, Francisco Gama da Silva' ${ }^{1}$, Humberto J. de Medeiros ${ }^{1}$, Angelo G. Roncalli ${ }^{2}$ y Maria I. Knackfuss ${ }^{2}$ 1 Departamento de Educação Física. Universidade do Estado do Rio Grande do Norte - UERN,
joaobatista@uern.br, franciscogama.gama@bol.com.br, hjmbeto@bol.com.br

2 Universidade Federal do Rio Grande do Norte-UFRN. roncalli@ufrnet.br, mik@ufrnet.br

Recebido em 24 Julho 2008/Enviado para Modificação 29 Dezembro 2008/Aprovado 11 Janeiro 2009

RESUMO

Objetivo O objetivo deste estudo é descrever o estado nutricional de crianças com idade entre 10 a 17 anos matriculados em escolas públicas e privadas de diferentes regiões com características específicas do semi-árido brasileiro.

Material e métodos Estudo transversal do tipo descritivo e comparativo, com uma amostra probabilística estratificada $n=1701$ (900 homens e 801 mulheres), escolhidas intencionalmente em três cidades das regiões Leste Potiguar $n=1 \quad 158$; Oeste Potiguar $n=312$; e Central Potiguar $n=231$, divididos em três grupos etários de 10 a 12 anos, 13 e 14 anos e $\geq 15$ anos. Para a classificação do índice de massa corporal, foi utilizada os pontos de corte de Conde e Monteiro.

Resultados Entre as regiões existe uma diferença significativa com $p<0001$, na distribuição do índice de massa corporal. A RLP apresentou um excesso de peso e obesidade (16,8 \% e 15,2 \%), a ROP (16,3 \% y $9,6 \%$ ) e a RCP $(10,4 \%$ e $3,9 \%)$ respectivamente.

Conclusões Adolescentes das regiões mais favorecidas, especialmente os estudantes pertencentes às escolas privadas do estado, tem uma prevalência de sobrepeso e obesidade.

Palavras Chave: Transição nutricional, transtornos nutricionais, estudos epidemiológicos, saúde pública (fonte: DeCS, BIREME).

\section{ABSTRACT}

Objective This study was aimed at describing the nutritional status of 10 to 17 year old children enrolled in public and private schools from different regions having specific Brazilian semi-arid area characteristics.

Material and methods This was a cross-sectional, descriptive and comparative study using a stratified probability sample $(n=1701) .900$ children were male and 801 female selected from three cities, randomly chosen from 3 cities which were representative of the regions: Leste Potiguar-RLP $(n=1,158)$, Oeste Potiguar-ROP $(n=312)$ and Central Potiguar-RCP $(n=231)$. They were divided into three age groups: 
10 to 12 years, 13 and 14 years and $e \geq 15$ years. Conde and Monteiro cutoff points were used for body mass index (BMI) classification.

Results Regarding the regions, there was a significant difference $(p<0001)$ in BMI distribution. RLP presented excess weight and obesity (6.8\%, $15.2 \%)$, ROP (16.3 $\%, 9.6 \%)$ and RCP (10.4\%, $3.9 \%)$, respectively.

Conclusions Adolescents in the most favored regions (especially students in private schools in the region) had a prevalence of being overweight and suffering from obesity.

Key Words: nutritional transition, nutrition disorder, epidemiologic study, public health (source: $\mathrm{MeSH}, \mathrm{NLM}$ ).

\section{RESUMEN}

Estado Nutricional de los estudiantes de la región semiárida del noreste de Brasil

Objetivo El objetivo de este estudio es describir el estado nutricional de los niños de 10 a 17 años matriculados en escuelas públicas y privadas de diferentes regiones brasileñas semi-áridas.

Material y métodos Estudio transversal, descriptivo y comparativo, con una muestra probabilística estratificada conformada por 1701 escolares (900 varones y 801 mujeres), elegidos al azar en tres ciudades, representativas de regiones: Leste Potiguar-RLP, $n=1158$; Oeste Potiguar-ROP, $n=312$; Central y Potiguar-RCP, $n=231$, divididos en tres grupos de edad, 10 a 12 años, 13 y 14 años y $\geq 15$ años. Se usó el método de los puntos de corte para Conde y Monteiro para clasificar el índice de masa corporal.

Resultados Relativa a las regiones hubo una diferencia significativa con $p<0001$, en la distribución del índice de masa corporal. La RLP presenta exceso de peso y obesidad (16,8 \%, 15,2\%), el ROP (16,3\% y $9,6 \%$ ) y RCP (10,4 \% y $3,9 \%$ respectivamente,

Conclusiones Adolescentes en las regiones más favorecidas, especialmente los estudiantes pertenecientes a las escuelas privadas en el estado, tienen una prevalencia de sobrepeso y obesidad.

Palabras Clave: Transición nutricional, trastornos nutricionales, estudios epidemiológicos, salud pública (fuente: DeCS, BIREME).

$\mathrm{E}$ studos mostram que o excesso de peso é um malefício em países desenvolvidos. Em países em desenvolvimento há uma coexistência de doenças nutricionais, seja por carência alimentar, caracterizando a desnutrição, ou por excesso de alimentos, configurando um quadro de sobrepeso ou obesidade $(1,2)$, sendo esta última considerada como uma das principais ameaças à saúde no mundo. Sua presença na adolescência é um preditor significante de sua prevalência na fase adulta (3). 
No Brasil, com toda sua diversidade social, econômica e cultural, estimou-se que cerca de $40 \%$ dos indivíduos adultos do país apresentam excesso de peso, ou seja, IMC igual ou maior que $25 \mathrm{Kg} / \mathrm{m}^{2}$, não havendo diferença substancial entre homens e mulheres. Os dados referentes às crianças brasileiras, levantados em 1989 pelo Instituto Nacional de Alimentação e Nutrição (INAN) e pelo Programa Nacional de Saúde e Nutrição (PNSN), apontam que cerca de um milhão e meio de crianças são obesas, com maior prevalência nas meninas das áreas de maior desenvolvimento. No entanto, esse quadro está mudando, pois, o perfil dos indivíduos do sexo masculino das classes sociais menos favorecidas, caracterizam a denominada transição epidemiológica $(4,5)$.

Considerando os aspectos ambientais apontados como os fatores de maior contribuição no aspecto nutricional de populações, especialmente em crianças e adolescentes, observa-se a necessidade da realização de estudos regionalizados que enfatizem e abordem as especificidades e características dos contextos imediatos $(6,7)$. Este estudo tem como objetivo descrever o estado nutricional de escolares, matriculados em escolas públicas e privadas de diferentes regiões do estado Rio Grande do Norte, situado na região Nordeste do Brasil, que possuem características geográficas variadas e especificas do semi-árido brasileiro (8). Este estudo busca contribuir num melhor mapeamento do espectro nutricional dos adolescentes dessa região, colaborando no delineamento da emergente transição nutricional no Brasil e a implantação de políticas públicas $(9,10)$.

\section{MATERIAL E METÓDOS}

Estudo descritivo de corte transversal, com tipologia comparativa (11), com uma amostra probabilística de 1701 escolares, estratificada em relação ao sexo, idade e tipo de escola. Sendo 900 do sexo masculino e 801 do sexo feminino, na faixa etária dos 10 aos 17 anos, selecionados aleatoriamente, sendo todos matriculados em escolas públicas e privados na zona urbana de três cidades do estado do Rio Grande do Norte, que foram escolhidas de forma intencional por estarem situadas em regiões distintas do Estado e apresentarem características sóciogeográficas, diferenciadas, sendo; Natal na Região Leste Potiguar (RLP) n=1 158, ( $n=589$ masculino e $n=569$ feminino); Mossoró na Região Oeste Potiguar (ROP) $n=312$ ( $n=150$ sexo masculino e $n=162$ feminino) e; Lajes na Região Central Potiguar (RCP) $n=23$ ( $n=161$ masculino e $n=70$ feminino), sendo as duas primeiras de maior densidade demográfica do estado $(8,12,13)$.

Considerando a escola pública e privada na definição do status sócioeconômico, a amostra foi estratificada, tendo a RLP n=760 e n=398; na ROP 
n=205 e n=107; na RCP n=119 e n=112, respectivamente. Em relação à faixa etária, considerando os estágios de maturação biológica na adolescência (14) e os valores médios da idade estimados a partir dos estágios maturacionais específicos nessa população por De Medeiros ${ }^{1}$, que enfatiza um retardo em torno de dois anos nas médias da idade púbere das meninas quando comparados a outros estudos, definiu-se três categorias para meninos e meninas; 10 e 12 anos, 13 e 14 anos e e $\geq 15$ anos.

O número de sujeitos foi determinado por um estudo de tamanho amostral, após estudo piloto utilizando a equação $\mathrm{n}=((\mathrm{z} * \mathrm{~d}) / \mathrm{E})^{2}$, onde $\mathrm{n}=$ amostra, $\mathrm{Z}=$ intervalo de confiança $95 \%$, d=desvio padrão de estimativa, E=Erro padrão de estimativa (11). Alunos não autorizados por seus respectivos responsáveis, aqueles que apresentavam algum tipo de enfermidade ou deficiência e os que não se dispuseram em ser avaliados, não foram inseridos neste trabalho. A coleta de dados foi realizada de abril a outubro de 2007, por uma equipe treinada no Laboratório de Atividade Física e Saúde-LAFIS/UFRN, aprovada pelo Comitê de Ética do Hospital Universitário Onofre Lopes, da Universidade Federal do Rio Grande do Norte.

Foram coletadas as medidas antropométricas da estatura e a massa corporal, para calcular os indicadores do IMC, definidos a partir dos pontos de corte de Conde e Monteiro, que entre as referências internacionais e nacionais mostrouse como o referencial de maior sensibilidade para triagens do espectro nutricional emnossa população de crianças e adolescentes $(15,16)$, objetivando a identificação de baixo peso (BP) excesso de peso (EP) e obesidade (OB, sendo considerados como peso normal $(\mathrm{PN})$ os sujeitos situados entre o $\mathrm{BP}$ e o $\mathrm{EP}(7,17)$.

Para a medida da massa corporal foi utilizada uma balança portátil marca Plena, com capacidade para $150 \mathrm{~kg}$. Na avaliação da estatura utilizou-se um estadiômetro marca seca, modelo 206(18).

Através de uma análise descritiva do IMC por grupo amostral, empregaramse testes de médias pela análise dos intervalos de confiança bem como o teste Qui ${ }^{2}$ para se conhecer a associação e o nível de significância entre as variáveis desse estudo nas respectivas regiões.

${ }^{1}$ De Medeiros HJ. Antropometria, qualidades físicas básicas e a dermatoglifia de escolares através dos estágios maturacionais do estado do Rio Grande do Norte. Tese do grau de Doutor em Ciências da Saúde [2005]. Localiza-se na: Universidade Federal do Rio Grande do Norte, Natal. 


\section{RESULTADOS}

Pelo intervalo de confiança (IC), observa-se que peso e estatura da RLP diferem das demais regiões, o mesmo não sendo atribuído entre as ROP e RCP, pois, a estatura e o peso de ambas convergem-se, sendo que a ROP apresenta menor intervalo em relação à estatura e a $\mathrm{RCP}$ em relação ao peso. Em relação ao IMC, o IC nos mostra uma aproximação do intervalo entre a RCP e a ROP, havendo uma pequena convergência do limite superior desta ultima com o limite inferior da RLP.

Tabela 1. Distribuição das médias do peso corporal, estatura e do IMC por faixas etárias nas regiões

\begin{tabular}{|c|c|c|c|c|c|}
\hline \multirow{2}{*}{ Regiões } & \multirow{2}{*}{ Faixa etária } & \multirow{2}{*}{$\begin{array}{c}\mathrm{N} \\
\mathrm{IC} 95 \%\end{array}$} & \multicolumn{3}{|c|}{ Dados antropométricos } \\
\hline & & & Peso & Estatura & IMC \\
\hline \multirow{8}{*}{ Leste Potiguar } & \multirow{2}{*}{10 a 12 anos } & $n=625$ & $42,8 \pm 10,5$ & $1,49 \pm, 09$ & $19,2 \pm 3,46$ \\
\hline & & IC 95\% & $41,9-43,7$ & $1,48-1,49$ & $18,9-19,5$ \\
\hline & \multirow{2}{*}{13 a 14 anos } & $n=402$ & $52,9 \pm 11,4$ & $1,62 \pm, 36$ & $20,5 \pm 3,69$ \\
\hline & & IC 95\% & $49,5-52,2$ & $1,59-1,66$ & $20,1-20,9$ \\
\hline & \multirow{2}{*}{$\geq 15$ anos } & $n=131$ & $57,4 \pm 11,6$ & $1,64 \pm, 08$ & $21,1 \pm 3,46$ \\
\hline & & IC $95 \%$ & $55,1-59,7$ & $1,63-1,66$ & $20,5-21,9$ \\
\hline & \multirow{2}{*}{ Total } & $\mathrm{n}=1158$ & $47,7 \pm 12,3$ & $1,55 \pm, 23$ & $19,8 \pm 3,61$ \\
\hline & & IC $95 \%$ & $46,9-48,4$ & $1,53-1,56$ & $19,6-20,1$ \\
\hline \multirow{8}{*}{ Oeste Potiguar } & \multirow{2}{*}{10 a 12 anos } & $n=195$ & $40,5 \pm 10,5$ & $1,46 \pm, 08$ & $18,8 \pm 3,75$ \\
\hline & & IC 95\% & $39,1-42,0$ & $1,45-147$ & $18,3-19,4$ \\
\hline & \multirow{2}{*}{13 a 14 anos } & $n=108$ & $47,9 \pm 9,8$ & $1,55 \pm, 08$ & $19,9 \pm 3,41$ \\
\hline & & IC $95 \%$ & $46,1-49,8$ & $1,53-1,57$ & $19,3-20,6$ \\
\hline & \multirow{2}{*}{$\geq 15$ anos } & $\mathrm{n}=9$ & $57,7 \pm 11,1$ & $1,61 \pm, 08$ & $22,4 \pm 4,27$ \\
\hline & & IC $95 \%$ & $49,9-65,4$ & $1,54-1,67$ & $19,4-25,3$ \\
\hline & \multirow{2}{*}{ Total } & $n=312$ & $43,6 \pm 11,1$ & $1,49 \pm, 09$ & $19,3 \pm 3,71$ \\
\hline & & IC 95\% & $42,3-44,8$ & $1,48-1,51$ & $18,9-19,7$ \\
\hline \multirow{7}{*}{ Central Potiguar } & 10 a 12 anos & $n=138$ & $\begin{array}{r}38,8 \pm 8,4 \\
374-403\end{array}$ & $\begin{array}{l}1,46 \pm, 07 \\
1,44-1,47\end{array}$ & $\begin{array}{l}18,1 \pm 2,66 \\
177-186\end{array}$ \\
\hline & \multirow{2}{*}{13 a 14 anos } & $\begin{array}{l}n=70 \\
n=85 \%\end{array}$ & $\begin{array}{c}3,4-40,3 \\
47,3 \pm 9,1\end{array}$ & $\begin{array}{l}1,44-1,4 l \\
1,57 \pm, 09\end{array}$ & $19,0 \pm 2,31$ \\
\hline & & IC $95 \%$ & $45,0-49,5$ & $1,55-1,59$ & $18,4-19,6$ \\
\hline & \multirow{2}{*}{$\geq 15$ anos } & $n=18$ & $52,4 \pm 7,2$ & $1,63 \pm, 06$ & $19,6 \pm 2,08$ \\
\hline & & IC $95 \%$ & $48,1-56,7$ & $1,59-1,67$ & $18,4-20,9$ \\
\hline & \multirow{2}{*}{ Total } & $\mathrm{n}=231$ & $42,2 \pm 9,7$ & $1,50 \pm, 10$ & $18,5 \pm 2,56$ \\
\hline & & IC $95 \%$ & $40,9-43,5$ & $1,49-1,52$ & $18,1-18,8$ \\
\hline
\end{tabular}

MC=Índice de massa corporal, $n=$ Total de sujeitos, IC=Índice de correlação

Observa-se um decréscimo das taxas de obesidade com o avançar da idade na RLP e na RCP, não ocorrendo essa mesma uniformidade na ROP, pois, a partir da faixa etária dos 15 anos há índices de maior expressão que nas faixas anteriores para as meninas. No outro extremo, o que chama a atenção no baixo peso é o crescimento em seus índices com o avançar da idade na RCP, enquanto nas demais observamos uma diminuição nas taxas de BP. Em relação ao excesso de peso observa-se um decréscimo significativo no avançar da idade na ROP e na RCP, enquanto na RLP encontramos valores em torno dos 9,0\%. Considerando a exceção feita inicialmente a ROP em relação à obesidade, há de se 
ressaltar que nas demais regiões ocorrem os maiores índices de excesso de peso e obesidade entre os 10 e 12 anos, passando a diminuírem com o aumento da idade. Apesar do aumento considerável com o avançar da idade em relação ao baixo peso na RLP, o que chama a atenção são os seus menores índices encontrados dentro das faixas etárias quando comparado às demais regiões.

As escolas privadas contribuem significativamente na prevalência do sobrepeso e obesidade nas RLP e ROP, com $p<0,003$ e $p<0,001$ respectivamente. Sendo que a RLP tem valores de $15,1 \%$ de sobrepeso nas escolas públicas e $19,8 \%$ nas escolas privadas, enquanto a ROP tem $13,2 \%$ e $22,4 \%$ e a RCP apresenta valores similares nas respectivas escolas de $10,1 \%$ e $10,7 \%$. Com relação à obesidade a RLP tem 13,6\% em escolas públicas e 18,3\% nas privadas. Sendo esses valores na ROP de $5,4 \%$ e $17,8 \%$ e na RCP de $1,7 \%$ e $6,3 \%$ respectivamente.

Tabela 2. Distribuição da classificação do IMC por região e tipo de escolas IMC=índice de massa corporal; $n=$ Total de sujeitos

\begin{tabular}{|c|c|c|c|c|c|}
\hline \multirow{2}{*}{ Regiões } & \multirow{2}{*}{ IMC } & \multirow{2}{*}{$N$} & \multicolumn{2}{|c|}{ Escolas } & \multirow{2}{*}{ Total } \\
\hline & & & Pública & Privada & \\
\hline \multirow{9}{*}{ Leste Potiguar } & \multirow[t]{2}{*}{ Baixo Peso } & $\mathrm{n}$ & 8 & 5 & 13 \\
\hline & & $\begin{array}{c}\% \\
\mathrm{n}\end{array}$ & $\begin{array}{r}1,1 \\
534\end{array}$ & $\begin{array}{r}1,3 \\
241\end{array}$ & $\begin{array}{r}1,1 \\
775\end{array}$ \\
\hline & Peso Normal & $\%$ & 70,3 & 60,6 & 66,9 \\
\hline & \multirow{2}{*}{ Excesso Peso } & $\mathrm{n}$ & 115 & 79 & 194 \\
\hline & & $\%$ & 15,1 & 19,8 & 16,8 \\
\hline & \multirow{2}{*}{ Obesidade } & $n$ & 103 & 73 & 176 \\
\hline & & $\%$ & 13,6 & 18,3 & 15,2 \\
\hline & \multirow{2}{*}{ Total } & $n$ & 760 & 398 & 1158 \\
\hline & & $\%$ & 100,0 & 100,0 & 100,0 \\
\hline \multirow{10}{*}{ Oeste Potiguar } & \multirow{2}{*}{ Baixo Peso } & $n$ & 5 & 2 & 7 \\
\hline & & $\%$ & 2,4 & 1,9 & 2,2 \\
\hline & \multirow{2}{*}{ Peso Normal } & $\mathrm{n}$ & 162 & 62 & 224 \\
\hline & & $\%$ & 79,0 & 57,9 & 71,8 \\
\hline & \multirow{2}{*}{ Excesso Peso } & $\mathrm{n}$ & 27 & 24 & 51 \\
\hline & & $\%$ & 13,2 & 22,4 & 16,3 \\
\hline & \multirow{2}{*}{ Obesidade } & $\mathrm{n}$ & 11 & 19 & 30 \\
\hline & & $\%$ & 5,4 & 17,8 & 9,6 \\
\hline & \multirow{2}{*}{ Total } & $\mathrm{n}$ & 205 & 107 & 312 \\
\hline & & $\%$ & 100,0 & 100,0 & 100,0 \\
\hline \multirow{9}{*}{ Central Potiguar } & \multirow{2}{*}{ Baixo Peso } & $\mathrm{n}$ & 1 & 3 & 4 \\
\hline & & $\%$ & ,8 & 2,7 & 1,7 \\
\hline & \multirow{2}{*}{ Peso Normal } & $\mathrm{n}$ & 104 & 90 & 194 \\
\hline & & $\%$ & 87,4 & 80,4 & 84,0 \\
\hline & \multirow{2}{*}{ Excesso Peso } & $n$ & 12 & 12 & 24 \\
\hline & & $\%$ & 10,1 & 10,7 & 10,4 \\
\hline & \multirow{2}{*}{ Obesidade } & $n$ & 2 & 7 & 9 \\
\hline & & $\%$ & 1,7 & 6,3 & 3,9 \\
\hline & Total & $\mathrm{n}$ & 119 & 112 & 231 \\
\hline
\end{tabular}

IMC=índice de massa corporal; $\mathrm{n}=$ Total de sujeitos 
Observou-se uma aproximação de valores entre meninas e meninos da RLP na ordem 15,8 \% e 14,6 \%, em relação à obesidade, que se apresenta na ROP com valores superiores nas meninas de $11,1 \%$ contra $8,0 \%$ dos meninos. Já na RCP há uma superioridade mais significativa na ordem de 8,6 e 1,9 respectivamente. $\mathrm{O}$ excesso de peso apresenta uma superioridade na RLP dos meninos com $20,9 \%$ e as meninas com $12,5 \%$. Ocorre, também, uma superioridade do EP dos meninos na RCP com 11,8 \% e 7,1\% nas meninas. Os valores do EP entre meninos e meninas na ROP mostram-se similares, com 16,7 \% e 16,0 \%, respectivamente. Há uma aproximação, mas, uma superioridade na ROP por parte dos meninos em relação ao peso normal com 72,7\% contra 71,0\% das meninas. Entre a RLP e a RCP percebemos uma inversão dos índices do PN entre os gêneros, mas, com uma manutenção similar da diferença, sendo meninos com $64,0 \%$, meninas com $69,9 \%$ e 85,7 \%, 80,0 \% respectivamente. Em relação ao BP, nota-se uma maior taxa no sexo masculino em todas as regiões. Sendo observado o valor de $p<0,05$ entre os gêneros em nossa amostra.

Tabela 3. Distribuição da classificação do IMC por regiões

\begin{tabular}{|c|c|c|c|c|c|}
\hline \multirow[b]{2}{*}{ Classificação IMC } & \multirow{2}{*}{$\begin{array}{l}\mathrm{n} \\
\%\end{array}$} & \multicolumn{3}{|c|}{ Regiões } & \multirow[b]{2}{*}{ Total } \\
\hline & & $\begin{array}{r}\text { Leste } \\
\text { Potiguar }\end{array}$ & $\begin{array}{r}\text { Oeste } \\
\text { Potiguar }\end{array}$ & $\begin{array}{l}\text { Central } \\
\text { Potiguar }\end{array}$ & \\
\hline \multirow{2}{*}{ Baixo Peso } & $n$ & 13 & 7 & 4 & 20 \\
\hline & $\%$ & 1,1 & 2,2 & 1,7 & 1,2 \\
\hline \multirow{2}{*}{ Peso Normal } & $n$ & 775 & 224 & 194 & 1196 \\
\hline & $\%$ & 66,9 & 71,8 & 84,0 & 70,3 \\
\hline \multirow{2}{*}{ Excesso Peso } & $n$ & 194 & 51 & 24 & 254 \\
\hline & $\%$ & 16,8 & 16,3 & 10,4 & 14,9 \\
\hline \multirow{2}{*}{ Obesidade } & $n$ & 176 & 30 & 9 & 231 \\
\hline & $\%$ & 15,2 & 9,6 & 3,9 & 13,6 \\
\hline \multirow{2}{*}{ Total } & $n$ & 1158 & 312 & 231 & 1701 \\
\hline & $\%$ & 100,0 & 100,0 & 100,0 & 100,0 \\
\hline
\end{tabular}

Observase uma diferença significativa entre as regiões do $\mathrm{RN}$, com o valor de $p<0,001$ na distribuição dos índices de classificação do IMC. Observamos uma aproximação das RLP e ROP em relação ao excesso de peso, tendo a RLP um maior índice de obesidade, seguida da ROP e por ultimo a RCP, que ao contrario da RLP detém os maiores índices de baixo peso e peso normal.

\section{DISCUSSÃO}

Observase nos resultados que ocorre uma adoção inversa dos índices de classificação entre as RLP e a RCP, onde a primeira detém os extremos com uma maior taxa de obesidade e menor taxa de peso normal, enquanto a segunda assume uma maior taxa de peso normal e a menor taxa de obesidade. O baixo 
peso se mostra muito próximo entre a ROP e a RCP. Há de considerarmos valores de sobrepeso similares nas ROP e RLP significantes. Em relação a outros estudos desenvolvidos no Brasil na mesma faixa etária (5), os valores de sobrepeso e obesidade da RLP se aproximam dos mesmos, podendo-se dizer o mesmo da ROP somente em relação ao sobrepeso.

Justifica-se esse aspecto pelas diferenças nos níveis sócio-econômicos das regiões, que se distinguem geograficamente (8), sendo a RLP privilegiada com um clima que vai de úmido a sub-úmido, seguida da ROP com clima sub-úmido seco e a RCP com características de semi-árido rigoroso. Os dados de peso, estatura e IMC dão indícios de que essas variáveis ambientais influenciam o aspecto nutricional dos adolescentes (19), pois, ao observarmos as variáveis antropométricas dos sujeitos da amostra em suas respectivas regiões, os índices de correlação mais significativos são na RLP, seguidos pela ROP em relação ao peso corporal e IMC, sendo superada pela RCP na estatura.

Conforme Malina (20) e Bouchard (6), quanto aos índices de contribuição de aspectos genéticos e fatores ambientais, o crescimento corporal tem maior influência genética, enquanto que o espectro nutricional, que envolve o peso corporal que pode resultar em índices que vão da desnutrição a obesidade, tem baixa relação com o genótipo e uma alta influência dos fatores ambientais.

Considerando a estratificação sócio-econômica em escolas públicas e privadas, notamos que as últimas contribuem significativamente nas três regiões para prevalência de sobrepeso/obesidade. Percebe-se que há uma inversão nos valores, onde, as escolas privadas da ROP contribuem mais para o sobrepeso enquanto as escolas privadas da RLP assumem esse papel na obesidade (12). Esses dados mostram tendências similares encontradas por Campos (21), ao analisar a relação entre o nível socioeconômico e a prevalência de sobrepeso e obesidade em adolescentes escolares do ensino público e privado do Município de Fortaleza, a partir do índice de massa corporal, utilizando Critérios de Classificação Econômica do Brasil, observando uma maior prevalência de sobrepeso/obesidade nas classes de melhor nível econômico.

Em relação à idade, percebe-se nas três regiões que ocorre um decréscimo gradativo com o avançar da mesma, exceção da obesidade por parte das meninas na ROP, onde ocorre uma aproximação dos índices na primeira e na segunda faixa etária. As meninas das regiões menos favorecidas apresentaram um retardo maturacional, pois, observa-se um decréscimo quase unânime dos índices de sobrepeso/obesidade entre as faixas etárias nas três regiões, os demais valores 
apresentam à mesma ordem decrescente, também relatado por Silva (22), que verificou a prevalência de sobrepeso e obesidade em crianças e adolescentes em um estudo em diferentes condições socioeconômicas na cidade do Recife, detectando a prevalência do sobrepeso/obesidade maiores junto aos sujeitos de menor idade pertencentes às classes de melhor poder econômico.

De uma forma mais ampla, a prevalência entre os adolescentes é na ordem de $14,9 \%$ de sobrepeso e 13,6 \% de obesidade, distinguindo-se dos valores apontados por Abrantes (23) envolvendo adolescentes das regiões Nordeste e Sudeste do Brasil, onde tivemos respectivamente $9,9 \%$ e 1,8\%, com superioridade para as mulheres de menor idade nas classes de melhor poder aquisitivo. Essa distinção corrobora com outros estudos $(19,24)$ ao evidenciar um retardo maturacional das meninas nas regiões de menores condições sócio-econômicas, pois, em condições inversas, há uma maior probabilidade delas maturarem mais cedo, propiciando a produção dos hormônios androgênios em maior quantidade, caracterizando a maturação biológica dos adolescentes.

Ao analisarmos os resultados conclui-se que os adolescentes nas regiões mais favorecidas, especificamente os alunos pertencentes às escolas privadas no estado, apresentam uma predominância de sobrepeso e obesidade. Recomenda-se um aprofundamento dos aspectos multifatoriais do espectro nutricional em crianças e adolescentes desta população, considerando os fatores maturacionais e ambientais como definidores precoces do quadro nutricional na idade adulta

\section{REFERENCIAS}

1. Barreto SM, Pinheiro ARO, Sichieri R, Monteiro $C A$, Batista Filho M, Schimidt MI, et al. Análise da estratégia global para alimentação, atividade física e saúde, da Organização Mundial de Saúde. Epidemiol serv saúde. 2005;14(1):41-68.

2. Barría PRM, Amigo $\mathrm{CH}$. Transición Nutricional: una revisión del perfil latinoamericano. Arch latinoam nutr. 2006;56(1):3-11.

3. Bouchard C. Obesity in adulthood-the importance of childhood and parental obesity. N Engl J Med. 1997 Sep 25;337(13):926-7.

4. Batista Filho M, Rissin A. A transiçäo nutricional no Brasil: tendências regionais e temporais. Cad saúde pública. 2003;19(supl.1):S181-S91.

5. Fisber M. Atualização em obesidade na infância e adolescência. Atheneu. São Paulo; 2005.

6. Bouchard C, Perusse L. Genetic aspects of obesity. Ann N Y Acad Sci. 1993 Oct 29;699:26-35.

7. Conde WL, Monteiro CA. Valores críticos de Índice de Massa Corporal para classificação do estado nutricional de crianças e adolescentes brasileiros. J Pediatria. 2006;82(4):266-72.

8. IDEMA. Perfil do Estado do Rio Grande do Norte. [Internet]; Disponible em: www.idema.rn.gov.br. Consultado 28 de Junho de 2008. 
9. Monteiro CA, Mondini L, Souza ALMd, Popkin BM, Monteiro CA. Da desnutrição para a obesidade: a transição nutricional no Brasil. Velhos e novos males da saúde no Brasil: a evolução do país e de suas doenças. Säo Paulo:Hucitec;1995. p. 247-55.

10. Marchioni DML, Zaccarelli EM. Transição nutricional. Hig aliment. 2002;16(96):16-22.

11. Thomas JR, Nelson JK. Métodos de pesquisa em atividade física. $3^{\mathrm{a}}$ ed. Porto Alegre: Artmed; 2002.

12. Perestrelo JPP, Martins IS. Modernização rural: transformações econômicas e suas implicações demográficas, epidemiológicas e nutricionais nos municípios de Monteiro Lobato e Santo Antonio do Pinhal. Saúde Soc. 2003;12(2):38-55.

13. Oliveira AMA, Cerqueira EMM, Souza JS, Oliveira, AC. Sobrepeso e Obesidade Infantil: Influência de Fatores Biológicos e Ambientais em Feira de Santana, BA. Arq Bras Endocrinol Metab. 2003; 47 (2): 144-150.

14. Matsudo SMM, Matsudo, VKR. Validade da auto-avaliação na determinação da maturação sexual. Revista Brasileira de ciências e movimento. 19915(2); 18-35.

15. Vitolo MR, Campagnolo PDB, Barros ME,Gama CM,Lopez FA. Evaluation of two classifi cations for overweight among Brazilian adolescents. Rev Saúde Pública:. 2007;41(4):653-6.

16. Glaner MF. Índice de massa corporal como indicativo da gordura corporal comparado às dobras cutâneas. Rev Bras Med Esporte. 2005;11(4):243-6.

17. Monteiro POA, Victora CG, Barros FC, Tomasi E. Diagnóstico de sobrepeso em adolescentes: estudo do desempenho de diferentes critérios para o Índice de Massa Corporal. Rev Saúde Pública. 2000;34(5):506-13.

18. Guedes DP. Manual prático para avaliação em educação física. Barueri-SP: Manole; 2006.

19. Guedes DP. Crescimento, composição corporal e desempenho motor de crianças e adolescentes. São Paulo-SP; 1997.

20. Malina RM. Growth and Development. Minneapolis: Burges Publishing Company; 1975.

21. Campos LA, Almeida PC, Leite AJM. Socioeconomic status and its influence on the prevalence of overweight and obesity among adolescent school children in the city of Fortaleza, Brazil. Rev de Nutr. 2006; 19(5):531-8.

22. Silva GAP, Balaban G. Motta, MEFA. Prevalência de sobrepeso e obesidade em crianças e adolescentes de diferentes condições socioeconômicas. Rev Bras Saúde Matern Infant. 2005; 5 (1):53-9.

23. Abrantes MM, Lamounier JÁ, Colosimo EA. Prevalência de sobrepeso e obesidade nas regiões Nordeste e Sudeste do Brasil Rev. Associação Médica Brasileira. 2003; 49(2):162-6.

24. Oliveira CL, Mello MT,Cintra IP, FISBERG M. Obesity and metabolic syndrome in infancy and adolescence. Rev Nutr. 2004; 17(2):237-45. 